\section{The effect of a human facial context on the discrimination and recognition of curved lines*}

\author{
ANNE D. PICK, JACQUELINE J. HALES, MONICA D. CHRISTY \\ and GUSTI W. FRANKEL \\ University of Minnesota, Minneapolis, Minn. 55455 \\ and \\ JOSEPH A. GLICK \\ City University of New York, New York, N.Y..10010
}

Children learned either to discriminate or to recognize curved lines which either represented mouths of face drawings or were surrounded by a plain square. The effect of the face context depended on the experimental task. Children who learned to discriminate among "sad" mouths made more errors in a transfer task than did children who learned to discriminate among unrepresentative lines. In the recognition task, direction of curvature of the critical lines, and not their context, affected performance difficulty.

Improvement in discrimination almost certainly involves active search for and detection of distinctive features of the relevant stimulus array (Gibson, 1969). Among the variables which might reasonably be expected to influence the ease of detection of distinctive features of an array is the familiarity of the features. Thus, Gibson (1969, pp. 105-108) investigated the recognizability of cartoon-like drawings in two orientations. In one orientation, the drawings resembled profiles of human faces, and in the other orientation, they resembled unfamiliar script writing. Those drawings in the profile orientation were recognized with greater accuracy than were the same drawings in the writing orientation. The distinctive features were presumably easier to detect when the drawings were oriented such that the features could be identified as representations of facial features.

One variable which might affect the relation between familiarity of features and their detectability is the task which confronts the $\mathrm{S}$. The specific task requirements-to discriminate among objects present in view or to recognize and identify objects previously viewed-might lead Ss to attend to different features of an array or to store previously detected features in different ways. For example, the results of a study by Gliner, Pick, Pick, \& Hales (1969) suggest that when a task requires the identification of an object previously perceived, children attend more to the shape features than to the texture

*'This research was supported by a Program Project grant, No. 5P01HD05027, from the National Institute of Child Health and Human Development to the University of Minnesota. differences. Such a result may reflect the usefulness of shape information in specifying the identity of objects. If the task requires some process other than object identification, attention may be directed toward different aspects of the objects. The familiarity, or usual context, of the features may affect the way in which attention is directed for a particular task. Thus, for example, with the figures of the Gibson experiment, if the task had been one of describing the letters of the unfamiliar alphabet, the profile orientation might have inhibited rather than facilitated attention to the relevant features of the drawings.

The present investigation is an attempt to explore the effects of a meaningful or familiar context and the experimental task on the detection of distinctive features. The tasks used were discrimination and recognition. The meaningful context was a two-dimensional representation of a human face. The distinctive features were different degrees of curvature of lines either as mouths in the context of a face or as single lines in the absence of a meaningful context.

The initial experiment was a pilot study in which preschool children learned to discriminate among lines which were either mouths or contextless curved lines and which curved either upward or downward. Following the discrimination training, transfer conditions were introduced which differed from the training conditions in either the method of stimulus presentation (from simultaneous to successive) or the direction of curvature of the lines, or both. The results of this pilot study showed that changing the method of stimulus presentation for the transfer task made that second task extremely difficult. In addition, there was a clear tendency for children who were discriminating faces to make more errors during the early transfer trials than children who were discriminating curved lines without the face context. This suggestion of a possible interference effect from learning to discriminate mouths rather than lines without meaningful context led to the design of the first experiment.

\section{EXPERIMENT 1}

The purpose of this experiment was to investigate the effect of learning to discriminate mouths as opposed to contextless curved lines on a subsequent transfer task. The method of presentation (successive or simultaneous) was not manipulated in this experiment, since that variable was not of major interest.

Subjects

The Ss were 48 preschool children enrolled either in a university laboratory nursery school or in a Montessori nursery school. The children were assigned randomly to one of the three transfer groups.

\section{Materials}

The stimulus forms were mounted on $4 \frac{1}{2}$-in. gray cardboard squares. The faces stimuli were representations of heads. A 4-in.-diam white paper circle was the head, and a stylized black paper shape was affixed to the top of the circle for the hair. The eyes, nose, and mouth were drawn with india ink. The circle was divided into four equal quadrants, with the horizontal axis as the location for the eyes. The eyes were 1 -in. straight lines separated 1 in. apart. The nose and mouth were oriented about the vertical axis. The nose was a $1 / 2$-in. line with a slight negative curvature, $1 / 2$ in. down from the center point; the mouth was $1 \frac{1}{4}$ in. down from the center point. The standard mouth was a 1 -in. straight line, and all variations of curvature were restricted to this inch-long horizontal distance. Variation in curvature was specified in terms of the radius of curvature $r$ (in millimeters): $r$ $=100 / \mathrm{n}$, where $\mathrm{n}$ varied from 1 to 10 in unit steps. Thus, the mouth with the greatest curvature was a half-circle with a radius of curvature of $10 \mathrm{~mm}$, and the mouth that closely approached the straight-line standard mouth had a radius of curvature of $100 \mathrm{~mm}$. With this method, 21 possible variations of mouth curvature were generated with the mouths ranging from a half-circle negative curvature (a deep frown), denoted as Stimulus No. -10 , to the standard straight line, denoted as Stimulus No. 0 , to the half-circle positive curvature (a deep smile), denoted as Stimulus No. +10 . For all variations, the point on the curve of greatest deviation from a straight line ran through a point on the vertical axis, 


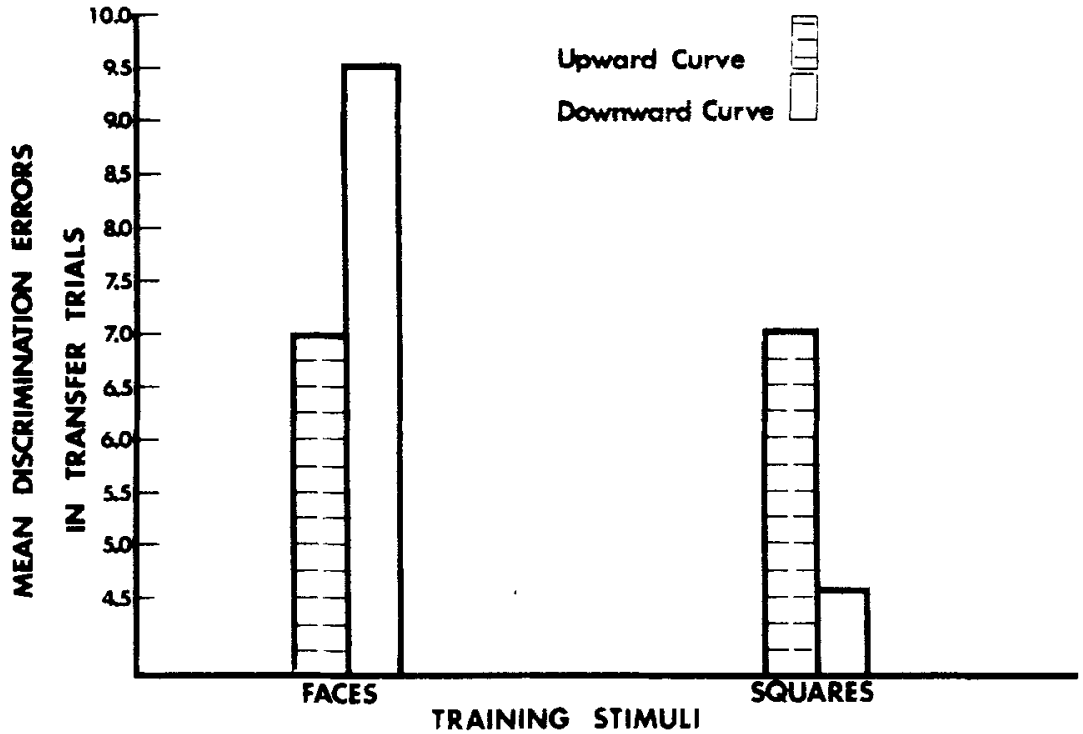

Fig. 1. Discrimination errors in transfer trials after training with different types of curved lines.

$11 / 4$ in. down from the center point.

The curved lines in the meaningless context were framed in otherwise empty squares (squares stimuli). The curves were drawn identical to the 21-step progression of the faces stimuli. Instead of a 4-in. circle, the stimulus was a 4 -in. white paper square, and the point of greatest curvature on the line was drawn through the intersection of the horizontal and vertical axes.

The stimuli were arranged in eight blocks of 10 trials each. For each block, one stimulus was designated as the standard. The standards were either the middle value of the series (i.e., \pm 5 ) or one or two steps on either side of the middle value. Within each block of 10 trials, the comparison forms included stimuli from one to four steps removed from the standard and either two or three stimuli which were identical to the standard. This range of stimulus differences was selected from pilot testing to provide differences which could not all be detected initially but most of which the children could learn to discriminate during the course of training. The arrangement of stimuli within the 10 trials was unsystematic. The first five blocks of trials were used for the training phase of the experiment and the remaining three were used for the transfer phase.

\section{Procedure}

Training. There were four subgroups of $S s$ for the training phase representing type of stimulus (faces or squares) and direction of curvature of the critical lines (up or down). The training procedure was the same for all Ss. The child was first seated at a table, shown some of the stimuli, and told that the game involved looking at the pictures very carefully to see which ones were alike and which were different. The $E$ then arranged the first 10 stimuli in two rows of 5 each in front of the child and placed the standard form above the rows. The child was told to look at the top (standard) picture very carefully, to look at each of the other (comparison) pictures in turn, and to say whether each comparison picture was exactly like or different from the top picture. For the first few trials, the $\mathrm{E}$ pointed to the critical lines to be compared. A correction procedure was used, and the standard form remained in place throughout the entire block. The child was encouraged to refer to the standard in making each judgment. When the child had made a judgment about all 10 comparison stimuli, the set was removed and replaced by the next standard and comparison stimuli. The trials continued until the child reached the criterion of 10 consecutive correct responses (excluding the one-step difference, which was too difficult for most children to learn). If criterion was achieved in the middle of a block, the block was completed so as not to disrupt the procedure for the child. The $E$ recorded the errors and the number of trials to criterion.

Transfer. The transfer phase was introduced immediately following the last training block. The child was told that he was playing the game very well and that the game would continue with different pictures. The procedure continued through the three transfer blocks, and the $\mathrm{E}$ responded with "O.K." or "Fine" to all of the child's judgments. When indicating the comparison to be made, the $E$ always pointed to the edge of the stimulus cards rather than to the critical lines.

There were three transfer conditions, which varied in the nature of the change in stimuli from the training to the transfer phase. For one group, the type of stimulus (faces or squares) was different from that used in training and the direction of curvature of the critical lines was also reversed. For a second group, the type of stimulus was also changed, but the direction of curvature of the critical lines was the same as it had been in training. For a third group, the type of stimulus was the same as it had been in training, but the direction of curvature of the lines to be discriminated was reversed. The $\mathrm{E}$ recorded the errors made during the transfer trials. Results

There were no differences among the groups in number of trials to criterion during the training phase. An analysis of the "same" errors (i.e., failures to judge a copy of the standard as "same") during the transfer phase revealed no pattern for this type of error.

Two three-way analyses of variance were performed on the data for discrimination errors during the transfer phase, one on the errors in the first transfer block and one on the errors in all three transfer blocks. The variables whose effects were assessed were: transfer condition, context (faces or squares) of stimuli during training, and direction of curvature of the critical lines during training.

The analysis of errors during the first 10 transfer trials revealed a significant Context by Direction of Curvature interaction $[F(1,36)=8.96$, $p<.01]$. Newman-Keuls tests showed that Ss trained on sad (i.e., downward curvature) faces made more errors ( $\bar{X}$ $=3.58$ ) in the early transfer trials than did Ss trained on downward curved lines which were not mouths $(\overline{\mathrm{X}}=$ 1.67). No main effects or other interactions from this analysis were significant.

The analysis of discrimination errors during all three transfer blocks ( 30 trials) revealed a significant effect of context $[F(1,36)=8.06, p<.01]$ and a significant Context by Direction of Curvature interaction $[F(1,36)=9.23$, $\mathrm{p}<.005]$. This interaction is illustrated in Fig. 1, in which it is apparent that the interaction clearly accounts for the main effect of context. Newman-Keuls tests showed that children who learned to discriminate sad mouths made significantly more errors $(\bar{X}=9.58)$ in the transfer trials than did either group of children who learned to discriminate curved lines which were not mouths $(\overline{\mathrm{X}}$ errors for smiling mouths $=6.92 ;$ for squares, upward 
curved lines $=7.08 ;$ for squares, downward curved lines $=4.67$ ). Thus, the trend which was revealed in the early transfer trials was enhanced during the entire transfer period-a trend toward greater difficulty regardless of transfer group for children who had been trained with sad faces. No other main effects or interactions were significant in this analysis.

The results of this experiment support the suggestion of the pilot study, that learning to discriminate lines in a meaningful context may inhibit subsequent performance with the same lines. Children who were trained to discriminate sad mouths made more errors in the transfer phase-regardless of their particular transfer group-than did children who were trained to discriminate contextless curved lines. In contrast, training in discriminating contextless curved lines transferred to subsequent discrimination of curved lines. There was no effect of the context or direction of curvature of the lines during transfer; the effects were dependent upon the conditions of training.

There is no obvious explanation for the fact that the context effect was specific to sad mouths and was not apparent for smiling mouths. One suggestion is that for these particular stimuli, the differences among smiling mouths might have been detected in a way unlike that for sad mouths. Specifically, the degree of curvature of smiles might have been assessed by comparing the curvature of a smile with the curve of the bottom of the face. This mode of solution of the initial discrimination task might produce greater transfer to the subsequent task than solution by assessing the absolute degree of "smile" or "sadness." It remains to be determined whether the difference in performance with sad mouths and smiles would also occur in a different type of task-a recognition task. The aspects of the stimulus assessed may be different for recognition and for discrimination. It should be noted that the differences between the sad mouths and the other stimuli were not differences in degree of difficulty in learning the initial discriminations. The differences had to do with the effect of initially learning to discriminate among sad mouths on subsequent discrimination of mouths or nonmouth curved lines.

The results thus far have shown an effect of learning a discrimination in a familiar context on subsequent discrimination performance. The second experiment was undertaken to determine whether the observed effect of context was general to another type of task-a recognition task.

\section{EXPERIMENT 2}

The purpose of this experiment was to investigate the effect of a familiar context on recognition of curved lines. The difficulty of recognizing mouths was compared with the difficulty of recognizing curved lines which were not mouths.

\section{Subjects}

The Ss were 44 children who were either attending summer art classes or were gathered in the home of a neighborhood friend. Their ages ranged from 5 to 8 years. The children were assigned to one of four stimulus conditions. The assignment was made unsystematically, except that the mean age and age range of each group was kept equivalent and the number of children from each source in each group remained the same.

$$
\text { Stimuli }
$$

The stimuli were those used in the previous experiment. They were arranged in five blocks of 10 trials each in the same manner as before. However, the particular arrangements of stimuli were different from those of the previous experiment.

\section{Procedure}

The four groups of Ss represented type of stimulus (faces or squares) and direction of curvature of the lines to be recognized. The training procedure was the same for all Ss. The child was seated at a table, shown some of the stimuli, and told that the game involved looking at one picture so that he could remember it when he saw some other pictures. The child looked at the standard form alone until he said that he was "ready," i.e., that he could recognize it when he saw the comparison forms. The $\mathrm{E}$ then covered the standard form and immediately showed the child the first five comparison forms. For each comparison form in turn, the child judged whether it was or was not identical to the standard form. After judging the first five comparison forms, the child again looked at the standard until he was "ready," and then was shown the second five comparison forms to judge. The succeeding blocks of trials were presented in the same manner as the first. The procedure continued through the five blocks of trials. No correction was made, but the child was encouraged to look at the standard as long as he wanted and to make his judgments with care. The $\mathrm{E}$ responded with "O.K." to all of the child's judgments. Each new block of trials was introduced with a comment about how well the child was playing the game. The $\mathrm{E}$ recorded each judgment. Results

The mean number of recognition errors made by $\mathrm{Ss}$ in each condition were as follows: square, upward curving lines, 17.9 ; square, downward curving lines, 12.8 ; face, smile (upward curve), 16.0; face, sad mouth, 13.1. A two-way analysis of variance was performed on the data for recognition errors during the five blocks of trials. The variables whose effects were assessed were context of the recognition forms and direction of curvature of the critical lines. This analysis revealed a significant effect of direction of curvature: $F(1,40)=4.71$, $p<.05$. Neither the effect of context nor the interaction was significant.

The effect of direction was that more errors were made in recognizing upward curving lines and smiles than in recognizing downward curving lines and sad mouths. This difference in difficulty of recognizing the two types of lines was without regard to the context.

When the results experiments are considered together, it is clear that the effect of the face context on the children's performance depends on the particular experimental task. Presumably, the task directs the child's attention differently to the critical stimulus lines. In the first experiment, the task required discrimination. Children who learned to discriminate among sad mouths made many errors in the transfer discrimination task. Learning to discriminate these lines as mouths in the face context made transfer of this discrimination difficult. What was learned seemed to be very specific to the mouth stimuli and not easily transferable to the other stimuli.

In the second experiment, the task required recognition of the curved line stimuli. In this case, the direction of curvature of the critical lines, and not their context, affected performance difficulty. Downward curving lines were more easily recognized than were upward curving lines.

One possible explanation can be suggested for the lack of effect of context on recognition. The upward curving lines may have been stereotyped as smiling mouths regardless of the presence or absence of E-imposed context. Many children referred to these lines as smiles, even in the "square" context, i.e., when the lines did not represent mouths. Perhaps, looking at a "smile," as opposed to looking at a downward curving line, makes it difficult to attend to varying degrees of a smile. Looking at smiling mouths may be an example of categorical perception. If all upward curved lines were treated as smiles, and if smiles were attended to categorically, then the high number of recognition errors for smiles as 
compared to downward curved lines may be reasonable. On the other hand, when the task was one of discrimination learning, the child was forced to attend to differences between smiles in order to reach the training criterion. In that case, the child may have learned the discriminations in a way that increased the generality of what he learned.

In summary, the results of these experiments provide evidence that the context in which a stimulus is presented and the task that one is asked to perform with that stimulus affect the perception and memory of it. Such effects are demonstrated here with a very simple stimulus form. The difficulty of performing tasks with more complex stimuli may depend even more specifically on the context in which those stimuli are presented.
REFERENCES

GIBSON, E. J. Principles of perceptua learning and development. New York: Appleton-Century-Crofts. 1969.

GLINER, C. R., PICK, A. D.. PICK, H. L. JR., \& HALES, J. J. A developmental investigation of visual and haptic preferences for shape and texture. Monographs of the Society for Research in Child Development, 1969,34(6. Serial No. 130). 